\title{
Schlussbemerkungen und Hinweise auf späte Dokumente
}

Diese Untersuchung machte auf die breite Tradition der Herstellung von Dokumenten der Andacht in Rollenform aufmerksam und brachte sie in erster Linie mit mystisch orientierten Gruppierungen unterschiedlicher Ausrichtung in Verbindung. Die ältesten erhaltenen Belegstücke stammen aus dem 14. Jh. Ihre herausragende Qualität lässt allerdings darauf schliessen, dass ihre Hersteller auf eine seit längerer Zeit gepflegte und damals durchaus etablierte Tradition hatten zurückgreifen können. Davon scheinen allerdings keine noch früheren Exemplare erhalten geblieben zu sein. Diese Feststellung trifft zumindest auf die handschriftlich angefertigten Exemplare zu.

Sowohl in formaler als auch inhaltlicher Hinsicht sind jedoch die in Tarš-Technik angefertigten Dokumente eng mit den in dieser Arbeit untersuchten handschriftlich angefertigten Rollen verwandt. Ihre Datierung ist zwar weiterhin mit grossen Unsicherheiten verbunden. K.R. Schaefer setzt aber einzelne Exemplare bereits früh an. ${ }^{1}$ Andere im Blockdruck-Verfahren angefertigte Belegstücke wurden auf Auktionen angeboten und datieren ebenso bereits aus früher Zeit (12. Jh.). Besonders wichtig ist diesbezüglich ein datiertes Exemplar, das im Jahr 535/1140 angefertigt wurde und mit Khorasan (No-Iran, Afghanistan, Usbekistan) in Verbindung gebracht wird. ${ }^{2}$ Sollten diese zeitli-

1 Schaefer, Enigmatic charms 5, 7 und 39: Das BlockdruckVerfahren soll in der islamischen Welt zwischen 9oo und 1430 bekannt gewesen sein. Nach 1400 soll es nach und nach verschwunden sein.

2 Das Belegstück stammt aus der Seldschukenzeit (Khorasan, datiert 535/1140): http://www.christies.com/LotFind er/lot_details.aspx?intObjectID $=5302917$ (Stand 20. Oktober 2017: ohne Abbildung; Stand 15. Oktober 2015: mit Abbildung). Siehe dazu die Beschreibung in Kapitel 7.3.

Man vergleiche damit auch: Sotheby's, Arts of the Islamic World, London, 6. Oktober 2010. Lot 38: „A Talismanic scroll, Khurasan, Persia, 11th/12th century AD. Arabic manuscript on paper, the Bismillah at the head, with the chen und geographischen Bezüge zutreffen, könnten sie auch die hier bei der Erklärung der frühen Dokumente (14. und 15. Jh.) ins Auge gefassten Abhängigkeiten von den im Kontext des Mongolensturms aus Khorasan in grösserer Zahl nach Westen ziehenden Mystikern stützen.

Die vorliegende Darstellung stellte diese Tradition der handschriftlich angefertigten Rollen anhand von repräsentativen Einzelstücken vor und nahm, indirekt zumindest, zugleich eine grobe chronologische und geographische Zuordnung vor. Frühe Exemplare wurden mit dem Städtedreieck zwischen Konya, Tabriz und Bagdad in Verbindung gebracht; sie datieren aus dem 14. und 15. Jh. Diese Tradition fand später unter unterschiedlichen Vorzeichen eine Fortsetzung in verschiedenen Gegenden der islamischen Welt. Zuletzt standen einerseits Belegstücke im Vordergrund, die mutmasslich im 18., allenfalls in der ersten Hälfte des 19. Jh., in Iran, wahrscheinlich in Südiran (Provinz Fārs mit der Hauptstadt Shiraz), entstanden sind. ${ }^{3}$ Ein anderer Ast dieser Tradition fand im Osmanischen Reich eine Fortsetzung, wie sich anhand ausgewählter Belegstücke aus dem 17. Jh. aufzeigen liess. Diese letzteren Exemplare dürften im Zusammenhang mit den Auseinandersetzungen zwischen Habsburg-Österreich und dem Osmanischen Reich stehen, die im Entsatz von Wien im Jahr 1683 einen Höhepunkt erreichten. ${ }^{4}$ Ausserdem lassen sich gerade ab dem 18. Jh. auch Belegstücke nachweisen, die mutmasslich auf dem Indischen Subkontinent angefertigt worden sind.

attributes of Allah written in two columns beneath, magic squares drawn at the top and bottom, strengthened at the edges. 65.2 by $9.8 \mathrm{~cm}$." Siehe http://www.sothebys.com/fr/ auctions/ecatalogue/2010/arts-of-the-islamic-world-lio22 3/lot.38.html (Stand 20. Oktober 2017).

3 Vgl. dazu Kapitel 5, v. a. 5.4 und 5.5.

4 Vgl. Kapitel 6.

(C) TOBIAS NÜNLIST, 2020 | DOI:10.1163/9789004429154_009

This is an open access chapter distributed under the terms of the CC BY-NC-ND 4.o license. 
Ausgewählte Dokumente dazu werden sogleich anschliessend vorgestellt (Kapitel 7.1).

Diese Tradition erlöscht im 18. oder 19. Jh. in der islamischen Welt nicht plötzlich. Es lassen sich vielmehr bis ins 20. Jh. hinein weitere Belegstücke nachweisen. ${ }^{5}$ Es lässt sich allerdings feststellen, dass diese Tradition zunehmend abflacht. Zwar sind aus dieser späteren Zeit Belegstücke in grösserer Zahl erhalten geblieben. Es entsteht aber der Eindruck, dass die entsprechenden Exemplare in Bezug auf ihre Vitalität und Originalität deutlich hinter den früheren Dokumenten zurückbleiben. Auch diese Beobachtung würde die hier vertretene These stützen, dass derartige Dokumente in erster Linie im Umfeld von mystisch orientierten Gruppierungen entstanden sind. Der auf den Dokumenten aus späterer Zeit zu beobachtende Verlust an Originalität brächte indirekt den Bedeutungsschwund zum Ausdruck, mit dem sich die islamische Mystik ab dem 19. und v. a. im 20. Jh. konfrontiert sieht. ${ }^{6}$

Diese Bemerkungen bedeuten nicht, dass mystisches Gedankengut gänzlich aus den islamischen Gesellschaften verschwindet. Allerdings legen Inhalt und Aufbau dieser späteren Dokumente nahe, dass die traditionalistisch orientierte Theologie der 'ulamä' und weitere der Modernisierung verpflichtete Kreise in den islamischen Gesellschaften zunehmend die Oberhand gewinnen. Dies wiederum hätte dazu geführt, dass die nur allzu oft stark heterodox orientierten Auffassungen aus den Derwischorden auch ihren bis anhin angestammten Platz auf den Rollen verloren. Diese Feststellung wird hier bewusst sehr allgemein formuliert und wäre anhand vertiefter Abklärungen zu verifizieren.

Diese Beobachtungen sollen hier zum Abschluss anhand der Untersuchung von zwei miteinander eng verwandten Belegstücken verdeutlicht werde. Bei den weiteren Ausführungen ist dem Umstand Rechnung zu tragen, dass sich

5 Vgl. unten Anm. 43: Ms. or. 14101 aus der Staatsbibliothek zu Berlin.

6 Knysh, Islamic mysticism 1. auf den beiden Exemplaren keine Kolophone mit unmissverständlichen Angaben zu ihrer geographischen und chronologischen Zuordnung feststellen lassen. Die Gruppe der in der weiteren Darstellung ins Auge gefassten Dokumente dürfte zumeist aus dem 18., allenfalls dem 19. Jh. stammen. Sie dürften mit grosser Wahrscheinlichkeit in indischen Kontexten entstanden sein, wobei diese Zuordnung in der Forschung nicht abschliessend geklärt ist. Es fällt jedenfalls auf, dass mehrere Exemplare dieser Art aus britischen Institutionen stammen. Dies legt die Vermutung nahe, dass sie in der ehemaligen britischen Kronkolonie Indien entstanden sind. Diese Annahme lässt sich einstweilen aber nicht beweisen. ${ }^{7}$ Diese Art von Dokumenten wird im folgenden anhand von zwei Belegstücken aus Paris und Kopenhagen vorgestellt. ${ }^{8}$

\subsection{Paris, Bibliothèque Nationale de France, Arabe 571, und Kopenhagen, Royal Library, Cod. Arab. Add. 49}

Arabe 571: ${ }^{9} 6.1 \times 368.0 \mathrm{~cm}$. Das Dokument setzt sich aus sieben aneinander geklebten Streifen zusammen. Die Überlappungen zwischen den Streifen betragen jeweils ca. $5 \mathrm{~mm}$. Die Streifen messen: 1 . $55.0 \mathrm{~cm} ; 2.62 .5 \mathrm{~cm} ; 3.66 .0 \mathrm{~cm} ; 4.66 .0 \mathrm{~cm} ; 5.63 .0 \mathrm{~cm}$; 6. $51.0 \mathrm{~cm}$; das Dokument franst am Schluss aus. F. Déroche datiert diese Rolle ins 17. Jh. und zieht eine Entstehung in der Türkei in Betracht. Diese Zuordnungen sind m. E. nicht gesichert; eine Anfertigung im 18. Jh. in Indien dürfte wahrscheinlicher sein. Sehr dünnes, leicht bräunliches Papier;

7 Für Hinweise auf allfällige Belegstücke aus Indien siehe Bayani et al., The decorated word: Qur'ans of the 17th to 19th centuries 2, 276-277 (Nr. 72) und 28o-281 (Nr. 74).

8 Paris, BNF, Arabe 571; Kopenhagen, The Royal Library, Cod. Arab. Add. 49.

9 Abbildungen der gesamten Rolle sind zugänglich unter http://gallica.bnf.fr/ark:/12148/btvib8433295० (Stand 7 . August 2017); vgl. die Beschreibung dazu bei Déroche, Les manuscrits du Coran. Du Maghreb à l'Insulinde 109 (Nr. 495). 
Wasserzeichen fehlen. Das Siebmuster verläuft in der Rollenrichtung. Das für Arabe 5102 (vgl. Kapitel 5.4) verwendete Papier ist feiner als jenes von Arabe 571. Auf der Rückseite am Anfang (frühere) Signatureinträge: Suppl. ar. 148 / 8; Arabe 571; 1513 III.

Cod. Arab. Add. $49:{ }^{10} 5.2 \times 357.5 \mathrm{~cm}$ (fragmentarisch erhalten, Anfang verloren); aufbewahrt in einem Behältnis aus Elfenbein $(2.5 \times 6.2 \mathrm{~cm})$. I. Perho nimmt in seiner Beschreibung des Dokuments weder eine geographische noch eine chronologische Zuordnung vor.

Das Dokument Arabe 571 weist am Anfang derart auffällige Ähnlichkeiten mit Kopenhagen Cod. Arab. Add. 49 auf, dass diese beiden Belegstücke aus demselben Atelier stammen dürften. Die Schreibweise des $h \bar{a}$ in Allāh in der Basmala unterscheidet sich allerdings stark; so dürfte wohl nicht derselbe Schreiber für deren Abschrift verantwortlich sein. Auf beiden Dokumenten steht am Anfang ein Titelbalken mit der Überschrift zu

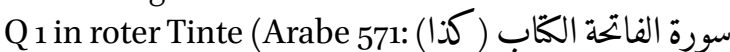
(مدنية وهي سبع آيات (Aarunter folgt die Abschrift von Q 1 in einem am Rand golden eingefassten Rechteck (schwarzes Nash). Es schliesst sich ein zweiter Titelbalken mit der Überschrift zu Q 2 an (Arabe 571: سورة البقرة مكية وهي مايتان ست سبع (كذا) نثاني يات ; darauf folgt der Beginn von Q 2 auf vier Zeilen in einem auf beiden Seiten golden eingefassten Rechteck.

Im Fall von Arabe 571 ist ein Anfangszierfeld erhalten geblieben (АВв. 161). Es enthält die Darstellung einer golden eingefassten Moscheekuppel, die blau ausgefüllt ist. Sie ist mit einem goldenen Aufsatz versehen. Seitlich ragen die beiden Verlängerungen des blauen Rahmens darunter in die Höhe. Sie können auch als Minarette interpretiert werden. Ein solches Anfangszierfeld fehlt auf Cod. Arab. Add. 49 aus Kopenhagen, da dessen Beginn verloren gegangen sein dürfte. Es ist gerade die Gestaltung der Kuppel auf Arabe 571, die eine Herstellung des Dokuments in Indien (bzw. Pakis-

10 Vgl. Perho, Catalogue of Arabic manuscripts III, 1271 (Cod. Arab. Add. 49). $\operatorname{tan)}$ als nicht ausgeschlossen erscheinen lässt. Ähnliche Kuppeln lassen sich z. B. in der SunehriMoschee in Lahore nachweisen. ${ }^{11}$ Moscheen aus dem 17. oder 18. Jh. aus dem Osmanischen Reich weisen flachere Kuppeln auf, als dies vorliegend der Fall ist. ${ }^{12}$ Mit den beiden Exemplaren aus Paris und Kopenhagen ist indirekt auch die Rolle Is 1620 (Chester Beatty Library, Dublin) zu vergleich. Auch sie weist am Anfang eine hoch aufragende Kuppel auf, wie sie aus Indien, nicht aber aus dem Osmanischen Reich bekannt sind. ${ }^{13}$ Diese Vergleiche mit Beispielen aus der Architekturgeschichte lassen eine Zuschreibung der soeben angeführten Exemplare (Arabe 571, Cod. Arab. Add. 49 und Is 1620) $\mathrm{zu}$ indischen Kontexten als wahrscheinlich erscheinen. ${ }^{14}$ Auch A.J. Arberry ordnet das Belegstück Is 1620 den Koranabschriften aus Indien zu. ${ }^{15}$

Der nächste Abschnitt der Rolle Arabe 571 misst ca. $20.5 \mathrm{~cm}$; auch er findet auf Cod. Arab. Add. 49 aus Kopenhagen eine Parallele (АВB. 162). Im Zentrum dieses Abschnitts steht eine säulenförmige Figur, die golden eingefasst und mit Text in schwarzem $\dot{G} u b \bar{a} r$ ausgefüllt ist. Auf der Basis ruht eine mandelförmige Figur, in deren Innern sich eine zweite mandelförmige Figur mit Text in rotem $\dot{G} u b a \bar{r}$ (Kopenhagen) befindet. Nach einer Verbreitung folgt gegen oben eine weitere mandelförmige Figur. Danach schliesst sich zuoberst der

11 Vgl. dazu https://archnet.org/sites/2752/media_conten ts/3499 und http://www.orientalarchitecture.com/sid/84 o/pakistan/lahore/sunehri-masjid (Stand beide 7. August 2017).

12 Man vergleiche damit z. B. die Nuruosmaniye-Moschee in Istanbul, die zwischen 1749 und 1775 errichtet wurde (https://archnet.org/sites/2011, Stand 7. August 2017).

$13 \mathrm{Zu}$ Is 1620 (Masse: $10.8 \times 737 \mathrm{~cm}$ ) vgl. Arberrry, Koran illuminated 75 (Nr. 238). Diese Rolle enthält eine Aufzählung der Čahārdah Mașūm in grosser Schrift; diese Aufzählung wird durch Text in kleinem $\dot{G} u b \bar{r}$ gebildet.

14 Man beachte auch die hochaufragende Kuppel des Šāh Čirāğ-Schreins in Shiraz; diese stammt in ihrer heutigen Form allerdings aus dem Jahr $195^{8}$ (vgl. https:// en.wikipedia.org/wiki/Shah_Cheragh). Siehe ausserdem: https://archnet.org/sites/4351/media_contents/95o und https://sacredsites.com/middle_east/iran/shiraz.html (Stand alle 13. Dezember 2017). 
Abschluss dieser säulenförmigen Figur an. Diese Säule wird zumeist von einem leeren Streifen eingefasst. Dem Rahmen dieses ersten Rechtecks entlang wurde der entstehende Leerraum mehrfach mit Textelementen in schwarzem und stellenweise rotem $\dot{G} u b \bar{a} r$ ausgefüllt. Auch der Rahmen auf der rechten und linken Seite des Dokuments wurde mit Text in $\dot{G} u b \bar{a} r$ ausgefüllt. Er ist hier diagonal ausgerichtet und verläuft von rechts unten nach links oben.

Auf dem Belegstück aus Kopenhagen (und ganz ähnlich auf jenem aus Paris) schliesst sich danach ein Rechteck von ca. $9.5 \mathrm{~cm}$ Höhe an. ${ }^{16}$ Es enthält im Mittelstreifen Text in schwarzem $\dot{G} u b \bar{a} r$ auf horizontalen Zeilen. Um dieses Rechteck im Innern herum sind Balken mit weiterem Text in $\dot{G} u b \bar{a} r$-Schrift angeordnet: der Text im oberen Balken wurde in rotem $\dot{G} u b \bar{a} r$ notiert. Der restliche Text in diesen Balken ist im Gegenuhrzeigersinn zu lesen und wurde schwarz kopiert.

Beide Rollen enthalten danach eine Abschrift des Thronverses (Q 2:255), ${ }^{17}$ der auf insgesamt 12 Textfelder verteilt in der Rollenrichtung kopiert worden ist (АВB. 163). Der Kunsthandwerker griff auf die bereits bekannte Technik zurück und füllte die durch Q 2:255 vorgegebenen Textelemente mit einem untergeordneten Text in schwarzem $\dot{G} u b \bar{a} r$ aus. Die folgende Übersicht stellt diese 12 Textfelder anhand des Belegstücks aus Paris vor:

Textfeld I: Basmala; ${ }^{18}$ mit Textfeld II beginnt die Abschrift von Q 2:255:الله لا اله الا هو: Text-

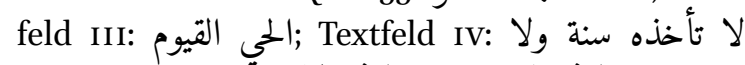
نوم; Textfeld v: له ما في السموات وما في الارم: Text-

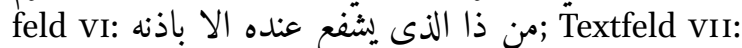
ولا يحيطون : يعلم ما بين ايديهم وما خلفهم

16 Ähnliches Vorgehen auch auf Is 1620 und Is Tf 99 aus der Chester Beatty Library, Dublin.

17 Dies ist ebenso auf Is Tf 99 der Fall; auf Is 1620 werden die Čahārdah Mașūm aufgeführt (Chester Beatty Library, Dublin).

18 Auch die Gestaltung dieses grossen Texts weist auf den beiden Exemplaren aus Kopenhagen und Paris auffällige Gemeinsamkeiten auf, ist aber nie völlig identisch; das alif in Allāh auf dem Belegstück aus Kopenhagen z.B. ohne das nach rechts absteigende Häkchen an der Spitze.

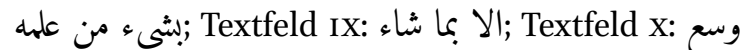

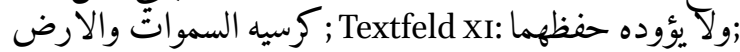
Textfeld XII: وهو العلى العظيه dem Pariser Belegstück werden durch je ein Rechteck voneinander getrennt, das durch jene Balken mit schräg ausgerichtetem Text eingerahmt wird, der im Gegenuhrzeigersinn zu lesen ist. Auf dem Pariser Exemplar schliessen sich ganz am Schluss nochmals zwei derartige Rechtecke an. Sie werden von einem längeren Rechteck unterbrochen, in dem sich erneut eine säulenförmige Figur erkennen lässt. Sie bildet offensichtlich das Gegenstück zur etwas längeren Figur am Rollenanfang. Auch hier am Schluss wird diese säulenförmige Figur durch Stellen in schwarzer $\dot{G} u b \bar{a} r$-Schrift gebildet. Diese beiden säulenförmigen Figuren erinnern gewissermassen an eine Stupa. Sollte diese Vermutung zutreffen, spräche auch diese Beobachtung für eine indische Herkunft des Dokuments. ${ }^{19}$ Allerdings ist dies einstweilen nicht gesichert. Ganz am Schluss von Arabe 571 steht übrigens Q 114, also die letzte Sure des Korans. Soweit ersichtlich handelt es sich auch bei den restlichen, auf diesem Exemplar kopierten Stellen ausschliesslich um Korantext. Die vorliegende Abschrift wurde allerdings nicht auf ihre Vollständigkeit hin überprüft.

Auch auf Cod. Arab. Add. 49 aus Kopenhagen wurde der Thronvers (Q 2:255) auf zwölf Textfelder verteilt kopiert, wobei die Aufteilung des Verses exakt mit jener auf dem Pariser Parallelstück übereinstimmt. Auf dem Belegstück aus Kopenhagen sind auch jene Rechtecke vorhanden, um die herum zusätzlich Balken mit weiteren Textelementen in $\dot{G} u b \bar{a} r$-Schrift angeordnet worden sind. Häufig wurde der Text im Innenfeld des Rechtecks gegenüber der Pariser Rolle auf modifizierte Art angebracht. Die auffälligste Abweichung zwischen Arabe 571 und Cod. Arab. Add. 49 besteht jedoch darin, dass das Belegstück aus Kopenhagen zusätzlich Abschnitte mit Zierfunktion aufweist. Auch in diesen Abschnitten wurden die Zierelemente mit Text in rotem und schwarzem $\dot{G} u b \bar{a} r$ ausgefüllt.

19 Derartige säulenförmige Elemente auch auf Is 1620 und Is Tf 99 (Chester Beatty Library, Dublin). 
Ein erstes solches Zierfeld wurde zwischen Textfeld I und II eingefügt. Es handelt sich um einen Doppelkreis, der aussen mit Text in schwarzem und innen mit Text in rotem $\dot{G} u b \bar{a} r$ ausgefüllt wurde (АВ B. 164). Im Innern des Kreises lassen sich zusätzlich sechs schmale, nach aussen weisende mandelförmige Blätter feststellen. Sie sind golden; an ihrer Spitze befinden sich im äusseren Ring des Kreises mit goldener Farbe ausgeführte blütenartige Zierelemente. Diese mandelförmigen Verzierungen lassen sich gerade auch auf abweichend ausgeführten Belegstücken aus dem Osmanischen Reich wiederholt feststellen. Ob die an anderer Stelle bereits geäusserte Vermutung zutrifft, diese Gestaltung könnte an eine von oben betrachtete Derwischmütze erinnern, muss offen bleiben. ${ }^{20}$

Zwischen Textfeld III und IV (Kopenhagen) folgt sodann ein weiteres Rechteck. Es enthält eine Blume mit einem Stiel aus Text in schwarzem und einer Blüte in rotem $\dot{G} u b a \bar{r}$ (АВв. 165). ${ }^{21}$ Zwischen Textfeld IV und v folgt ein weiteres Rechteck, das ähnlich aufgebaut ist wie seine Pendants auf dem Pariser Belegstück. Der einzige Unterschied besteht darin, dass der Text in schwarzem $\dot{G} u b \bar{a} r$ im Innenfeld nicht auf horizontalen Linien kopiert worden ist, sondern sich in einer golden eingefassten Kartusche befindet; die Zeilen in dieser Kartusche verlaufen in der Rollenrichtung.

Zwischen Textfeld v und vi (weiterhin Kopenhagen) folgt das nächste Rechteck mit einer Blume. Der Stiel und die Blätter werden durch Text in schwarzem $\dot{G} u b \bar{a} r$ gebildet. Die vier Blütenblätter wiederum sind golden eingefasst und mit Text in rotem $\dot{G} u b \bar{a} r$ ausgefüllt. An der Basis dieser vier Blütenblätter ist der $\dot{G} u b \bar{a} r$-Text schwarz. Zwischen Textfeld vi und viı schliesst sich das nächste Rechteck ohne gegenständliche Elemente an. Zwischen Textfeld VII und VIII folgt ein weiteres Rechteck. Es enthält einen goldenen Kreis, der durch sechs achsialsymmetrisch angeordnete und ebenso goldene Elemente in sechs Segmente auf-

20 Vgl. dazu Kapitel 5.3, vor Anm. 3 o.

21 Derartige Blumen ebenso auf Is Tf 99 (Chester Beatty Library, Dublin). geteilt wird. Diese sechs Kreissegmente wurden mit Text in schwarzem $\dot{G} u b \bar{a} r$ ausgefüllt. Zwischen Textfeld ViII und Ix folgt das nächste Rechteck ohne gegenständliche Elemente. Zwischen Textfeld IX und $\mathrm{x}$ befindet sich sodann wieder ein Abschnitt mit einer Blume. Stiel und Blätter sind erneut mit Text in schwarzem $\dot{G} u b \bar{a} r$ ausgefüllt. Die Blüte selbst entsteht durch Stellen in rotem $\dot{G} u b \bar{a} r$. Zwischen Textfeld x und XI schliesst sich das nächste Rechteck an, in dem der Text in mikroskopischer Schrift in geometrische Figuren eingepasst worden ist. Zwischen Textfeld XI und XII nochmals ein Rechteck mit einer Blüte. Sämtliche Blumen auf diesem Belegstück wurden anders gestaltet, einmal abgesehen davon, dass Stiel und Blätter stets schwarz, die Blüte selbst aber rot ausgefüllt worden sind. Nach Textfeld XII folgen nochmals zwei Rechtecke mit Textelementen in schwarzer Schrift. Zwischen diese beiden kleineren Rechtecke ist ein zusätzliches längeres Rechteck eingefügt worden. Es enthält eine säulenförmige Figur, deren Spitze auf das Ende der Rolle hinweist. ${ }^{22}$

Die Rolle aus Kopenhagen wird in einem Behältnis aus gelblich-weissem Elfenbein aufbewahrt. ${ }^{23}$ Es ist mit einem Deckel versehen, der das Behältnis oben verschliesst und in das die Rolle selbst hineinpasst, ohne über dessen oberen Rand hinauszuragen. Dies erschwert bzw. verunmöglicht es auch, die Rolle überhaupt wieder aus dem Behältnis herauszunehmen. Allerdings müssen sich auch die an der Herstellung dieses Dokuments beteiligten Kunsthandwerker dieses Problems bewusst gewesen sein. Sie lösten es, indem sie auch den Boden des Behältnisses als eigenständiges Element ausführten. So lässt sich das in diesem Zylinder enthaltene Dokument z. B. mit einem Bleistift oder dem Finger herausdrücken.

22 Eine ähnliche säulenförmige Figur ebenso auf Is Tf 99 (Chester Beatty Library, Dublin); siehe ebenso Is 1620 (Chester Beatty Library, Dublin).

23 Auch Is 1620 (Chester Beatty Library) befindet sich in einem Behältnis aus Elfenbein; die im folgenden festgehaltenen Besonderheiten aber nur beim Behältnis aus Kopenhagen. 
Dabei fällt zuerst der lose Boden heraus. Die Kunsthandwerker müssen äusserst sorgfältig gearbeitet haben, löst sich der Boden doch nur unter leichtem Druck. Solange man keinen Druck auf den Boden ausübt, meint man, ein normales Behältnis mit einem fest damit verbundenen Boden vor sich zu haben.

Aus dem 18. oder 19. Jh. sind zusätzliche Belegstücke bekannt, die oft ähnlich gestaltet sind, wie die beiden soeben näher vorgestellten Exemplare aus Paris und Kopenhagen. So ist die Existenz eines Dokuments aus der Biblioteca Apostolica Vaticana bekannt (Ms. Vat. Arab. 882), das in der Beschreibung dazu mit Indien in Verbindung gebracht und ins 18. Jh. datiert wird. ${ }^{24}$ Auf Is 1620 (Chester Beatty, Dublin) werden die Čahārdah Mașūm in Kartuschen aufgezählt. Am Anfang des Dokuments befindet sich eine hochaufragende Kuppel, was mutmasslichen auf einen indischen Ursprung hinweist. ${ }^{25}$ Eine ähnliche Gestaltung weisen zwei Dokumente aus Manchester, John Rylands Library, auf. ${ }^{26}$ Verschiedentlich lassen sich Hinweise darauf feststellen, dass derartige Belegstücke in spezialisierten Ateliers seriell angefertigt worden sind.

24 Orsatti, Le manuscrit islamique $278 \mathrm{f}$.

25 Kuppeln im Osmanischen Reich aus dem 18. Jh. sind flacher (vgl. dazu bei und in Anm. 12). Auf Is 1620 werden auch die Šuhadā’ fi Karbalä’ erwähnt, was im vornehmlich sunnitischen Osmanischen Reich ebenso überraschen würde wie die Erwähnung der Čahärdah Mașūm. Auf Is 1620 lässt sich am Anfang überdies eine stupa-artige Verzierung feststellen. Beim Feld für 'Alī alMurtad̄a fällt auf, dass die einzelnen Abschnitte des grossen Texts numeriert sind, was die Abfolge vorgibt, in welcher der darin enthaltene mikroskopische Text zu lesen ist.

26 Manchester, John Rylands Library, Arabic 9 und Arabic 10. Man vergleiche damit auch Cambridge, Fitzwilliam Museum: Sdearle, The illuminated manuscripts 167 (Nr. 148: 7. G. 8 - Burney. 3): „Amulet. An Himáyil or amulet containing the whole Corán, beautifully written in a small hand on a roll of paper $11 \mathrm{ft}$. $6 \mathrm{in}$. long and $3 \mathrm{in}$. wide. The text is arranged so as to form a pattern, and this read lengthwise forms the aiyat el kursi, Corán, Chap. 2, v. 256. It is contained in a little silver box of English make bearing the initials of the donor, Miss J.C. Burney, of Maida Hill, London, the sister of Col. Richard Burney. It was presented in $1861 . "$
Dieser Eindruck lässt sich bereits im Fall der beiden soeben vorgestellten Exemplare aus Paris und Kopenhagen nicht von der Hand weisen. In dieselbe Richtung weisen insbesondere auch die beiden Belegstücke Add. $5904^{27}$ und I.o. Islamic $4150^{28}$ aus der British Library. Sie stammen aus zwei unterschiedlichen Beständen der Sammlung, wie sich aus ihren Signaturen ableiten lässt. Bei der Konsultation liessen sich die beiden Belegstücke direkt nebeneinander legen. Beide Exemplare enthalten eine Abschrift des Thronverses (Q 2:255) in grossen Buchstaben, die mit Text in $\dot{G} u b \bar{a} r$ ausgefüllt sind. ${ }^{29}$ Die Einteilung der beide Belegstücke stimmt miteinander überein. Die Abweichungen zwischen den beiden Rollen bewegen sich im Millimeterbereich. Diese Beobachtung weist auf eine Herstellung in einer spezialisierten Werkstätte hin. Aufgrund der Signatur lässt sich zumindest im Fall von I.O. Islamic 415o A auf eine indische Provenienz schliessen. ${ }^{30}$ Bei der seriellen Produktion dieser Dokumente kamen mutmasslich auch Schablonen zum Einsatz. Sie gestatteten es, gerade den übergeordneten grossen Text (Q 2:255, Thronvers) rasch zu Papier zu bringen. Auch diese Hinweise auf eine serielle Herstellung der Dokumente deuten auf ein Abflachen der Tradition der Anfertigung derartiger Rollen hin.

27 London, British Library, Add. 5904; beschrieben in Cureton, Catalogus 57a (Nr. 29).

28 London, British Library, I.O. Islamic 4150; beschrieben in Storey, Catalogue of the Arabic Manuscripts in the Library of the India Office II.1: Qur'anic Literature $5 \mathrm{f}$.

29 Auch auf Tf 99 (Chester Beatty Library, Dublin) wurde der Thronvers (Q 2:255) auf Kartuschen verteilt kopiert; in diesem Fall entsteht der grosse Text ebenso durch eine Abschrift von Stellen in $\dot{G} u b a \bar{r}$. Das Dokument weist am Anfang weder Kuppel noch Anfangszierfeld auf. Zwischen den Kartuschen mit grossem Text wurden Blumen/Blüten und Mützen eingefügt. Vor dem Feld mit der Basmala eine Blume. Unmittelbar davor ging ein Abschnitt der Rolle verloren. Die stupa-artige Verzierung jedenfalls ist unvollständig. Diese Fehlstelle wurde mit zwei verschiedenen Papieren ausgebessert. 


\subsection{Hinweise auf weitere einer Untersuchung würdige Belegstücke}

Allerdings lassen sich auch in der späteren Zeit immer wieder Hinweise dafür finden, dass diese Tradition durchaus lebendig blieb und über eine gewisse Innovationskraft verfügte. Dies soll anhand ausgewählter weiterer Beispiele hier nochmals aufgezeigt werden.

Aus der Bodleian Library ist mit Ms. Arab. g. 14 $(\mathrm{R})^{31}$ ein Belegstück bekannt, dessen Layout grosse Ähnlichkeiten mit dem Dokument aus Kopenhagen (Cod. Arab. Add. 49) aufweist. Auch darauf ist der Thronvers (Q 2:255) auf die bereits geschilderte Art kopiert worden. Das auffälligste Merkmal dieses Exemplars besteht darin, dass es auf einem Stäbchen fixiert wurde, das sich im Innern einer gedrechselten Holzkapsel befindet. Dieser hölzerne Zylinder ist mit einem Schlitz versehen, aus dem der Anfang der Rolle herauslugt. Die Rolle lässt sich an dieser Zunge herausziehen. Am Schluss lässt sich das Dokument durch Drehen am Kapseldeckel wieder ins Innere seines Behältnisses befördern. Es ist ratsam, zuletzt einige Zentimeter aus dem Schlitz herausschauen zu lassen. Auch dieses Belegstück steht letztlich aber für das Abflachen der Tradition der Herstellung dieser Rollen. Die auf diesem Exemplar enthaltenen Elemente sind eigentlich alle von den bereits vorgestellten Paralleldokumenten aus Paris und Kopenhagen bekannt. Der originellste Aspekt dieses Belegstücks liegt - was wiederum bezeichnend ist - in seiner raffinierten Mechanik.

Es soll allerdings nicht verschwiegen werden, dass auch aus dieser späteren Zeit vereinzelt Dokumente erhalten geblieben sind, die eine eingehendere Auseinandersetzung damit als lohnenswert erscheinen lassen. Dazu zählt eine Rolle aus der Handschriftenabteilung der Universitätsbibliothek Teheran (Signatur 4662), die mutmasslich im 18. oder 19. Jh. entstanden ist. ${ }^{32}$ Dazu ist

31 Vgl. auch Laud. Or. Rolls g 2 (Bodleian Library, Oxford).

32 Siehe: Fihrist-i nusha-hā-i hatțīi Kitābhāna-i markaz̄̄i Dānišgāh-i Tihrān Ǧild 15, 76, s.v. „Ṭūmār“. Die Rolle ein Blatt aus einem Kodex aus der Khalili Collection mit zumindest teilweise identischem Inhalt bekannt. ${ }^{33}$ Auf dem Blatt aus dem Kodex ist der Inhalt aber in Spalten angeordnet; würde man das Blatt zerschneiden, könnte man die einzelnen Spalten bzw. Streifen zusammenkleben und daraus eine Rolle anfertigen. Das Heft mit dem erwähnten Blatt aus dem Kodex war anfänglich übrigens mit einer Koranhandschrift zusammengebunden. Dieses Heft ist jedoch zu einem späteren Zeitpunkt von der Koranhandschrift abgetrennt worden. ${ }^{34}$ Der Anlass dafür dürfte darin $\mathrm{zu}$ suchen sein, dass einem späteren Besitzer der Koranhandschrift die auf diesem Zusatzteil enthaltenen Elemente allzu suspekt erschienen. Er dürfte es deshalb vorgezogen haben, dieses zusätzliche Heft heraustrennen zu lassen. Dieser Schritt illustriert exemplarisch, wie die auf den Rollen oft enthaltenen heterodoxen Elemente ihre gesellschaftliche Akzeptanz und damit ihren „Sitz im Leben“ verlieren. Sie werden dabei auch physisch vom Koranexemplar getrennt. Auch das Kitābhāna-i Malik in Teheran besitzt mehrere Dokumente in Rollenform. Darunter befindet sich ein Prachtsexemplar, das dem Verfasser nach langem Verhandeln mit grossem Widerwillen ganz kurz gezeigt wurde. ${ }^{35}$

Tihrān, Dānišgāh, Ms. 4662, dürfte in der 2. Hälfte des 19 Jh. entstanden sein.

Codex Khalili Collection Accession no. Mss 412, fol. 1B; vgl. Maddison und Savage-Smith, Science, Tools, and Magic 58f.

34 Schriftliche Auskunft von Nahla Nassar, Khalili Collection, vom 14. August 2012: „I have just uploaded images of the 8 folios of mss 412 [...]. I am assuming you know that these folios were once attached to a Qur'an which is now also in the Collection?"

35 Dieses Exemplar enthält mehrfach Elemente heterodoxer Natur. Besuch im Kitābhāna-i Malik, Januar 2005; seine Signatur ist aktuell nicht mehr bekannt. Siehe für weiterführende Angaben: Afšār und Dānišpažū, Fihristi tartībì-i šumāra-i nusha-hā. Zīr-i nazar-i İ. Af̌sār wa M.T. Dānišpažū. Bā hamkārī-i Qudratullāh Pīšnamāzzāda. Tihrān, Kitābhuāna-i Millī-i Malik, 1372 h.s./1993 Staatsbibliothek zu Berlin, Signatur 4 A 40534-10, Ğild 10, $253(t \bar{u} m a \bar{r})$. 
Ein weiteres Belegstück, das hier nicht mehr eingehend untersucht wird, befindet sich im Pitt Rivers Museum (Oxford). ${ }^{36}$ Es enthält u.a. eine Aufzählung der 12 Imame. Am Anfang fällt überdies eine Verzierung auf, die sich in ähnlicher Form auf Dokumenten aus dem Umfeld der Bektāšiyya nachweisen lässt. ${ }^{37}$ Es sollen hier allerdings keine weiteren Vermutungen zur Einordnung dieses Dokuments angestellt werden.

Ebenso wenig wurden hier jene Dokumente auf Pergament untersucht, die in der Regel eine Aufzählung der Čahārdah Mașūm enthalten und somit aus einem schiitischen Umfeld stammen. Das beachtenswerteste Belegstück aus dieser Gruppe befindet sich in der Bayerischen Staatsbibliothek, München (Signatur Cod. arab. 2616), und ist zusammen mit seinem wohl ursprünglichen Behältnis erhalten geblieben. Es stammt wohl aus dem 19. Jh. ${ }^{38}$ Besonders viele Dokumente dieses Typs besitzt sodann die Bodleian Library in Oxford. ${ }^{39}$ Ein weiteres Exemplar dieser Art stammt ausserdem aus Basler Privatbesitz. ${ }^{40}$ Zwei weitere Belegstücke auf Pergament befinden sich in der Chester Beatty Library (Dublin). Bei KR 11 (Chester Beatty Library, Dublin) handelt es sich um ein Schutzdokument mit Gebe-

36 Pitt Rivers Museum, Oxford, Signatur: 1994.4.20.1-3 (siehe: objects.prm.ox.ac.uk/pages/PRMUID11107.html, Stand 20. Oktober 2017, mit Hinweisen auf Untersuchung durch den Verfasser am 22. Juli 2015): Breite des Dokuments: $7.8 \mathrm{~cm}$; Breite des Schriftspiegels: $6.6 \mathrm{~cm}$; Länge: $460.9 \mathrm{~cm}$. Zusammengesetzt aus mehreren Papierstreifen; zwischen den Abschnitten 1 und 2 scheint ein ganzer Streifen zu fehlen: Total: $460.9 \mathrm{~cm}$; die erhaltenen Streifen messen: $1.50 .2 \mathrm{~cm} ; 2.58 .3 \mathrm{~cm} ; 3.59 .6 \mathrm{~cm} ; 4.57 .9 \mathrm{~cm} ; 5$. $58.6 \mathrm{~cm} ; 6.57 .8 \mathrm{~cm} ; 7.58 .5 \mathrm{~cm} ; 8.60 .0 \mathrm{~cm}$.

37 Vgl. dazu de Jong, The iconography of Bektashiism, Plates 9, 12; aufrufbar unter http://www.islamicmanuscri pts.info/reference/articles/Jong-1989-Iconography-MME 4.PDF (Stand 19. Oktober 2017).

38 Vgl. dazu die Abbildungen unter http://daten.digitale -sammlungen.de/ db/ooo3/bsbooo39626/images/index .html?id=ooo39626\&groesser=\&fip=xsxswxseayaxdsyde ayaqrsen $\&$ no $=4 \&$ seite $=6$ (Stand 20. Oktober 2017).

39 Siehe Oxford, Bodleian Library, Ms. Arab. g. 34-41; neue Signatur: Or. Long. a. 21; insgesamt 8 Dokumente.

40 Im Besitz von Prof. Dr. R. Würsch, Basel. ten und Elementen aus dem Koran. Die soeben angeführten Dokumente auf Pergament dürften in ähnlichen sozio-religiösen Umfeldern entstanden sein, wie die hier vorgestellten Belegstücke auf Papier. ${ }^{41}$ Gestaltung und Inhalt von KR 9 (Chester Beatty Library), ebenso auf Pergament, unterscheiden sich aber deutlich von den weiteren hier untersuchten Belegstücken. KR 9 dürfte eine andere Funktion zukommen; es wird hier ausschliesslich der Vollständigkeit halber und wegen seines ausserordentlich hohen künstlerischen Werts erwähnt. ${ }^{42}$

Abschliessend soll nochmals auf ein Belegstück aus der Staatsbibliothek zu Berlin hingewiesen werden (Ms. or. $14101 ; 15 \times 486 \mathrm{~cm}$ ) ${ }^{43}$ Beim Beschreibstoff handelt es sich um weinrotes Papier aus industrieller Produktion. ${ }^{44}$ Es enthält in grosser goldener und silberner Schrift (Höhe ca. $10 \mathrm{~cm}$ ) die Schönen Namen Gottes. Sie werden durch eine entsprechende Anordnung von Koranstellen in $\dot{G} u b a \bar{r}$-Schrift gebildet. Gemäss den Angaben eines Händlers soll dieses Belegstück um 1970 in Syrien entstanden sein. ${ }^{45}$ Auch auf diesem jüngsten mir bekannten Dokument fehlen jene häufig heterodox geprägten Elemente, die sich auf den frühen Exemplaren mit grosser Regelmässigkeit feststellen liessen.

Diese Beobachtungen legen den Schluss nahe, dass das Rollenformat für Abschriften des Korans

41 Ein zusätzliches Belegstück aus Pergament ist abgebildet bei Maddison und Savage-Smith, Science, tools and magic, Part I: Body and Spirit 142 f. (Nr. 88).

42 KR 9: Dieses Dokument zeigt einen Mann und eine Frau, die aus Textstellen in $\dot{G} u b \bar{a} r$-Schrift gebildet werden. Funktion und Bedeutung dieses Belegstücks liessen sich noch nicht klären.

43 Die Rolle Ms. or. 14101 ist aufrufbar unter http://digital .staatsbibliothek-berlin.de/werkansicht?PPN=PPN8476 78849\&PHYSID=PHYS_ooo1\&DMDID=DMDLOG_ooo1 (Stand 19. August 2017).

44 Bei diesem Papier aus industrieller Produktion war es nicht mehr erforderlich, einzelne Streifen zusammenzukleben.

45 Mündliche Information von Ch. Rauch, Leiter der Orientabteilung, Staatsbibliothek zu Berlin, Januar 2009. Man beachte auch die Ursprungsangaben auf vier aufgeklebten Schildchen auf der Rückseite des Dokuments. 
vereinzelt bis in die jüngste Vergangenheit gepflegt wurde. Allerdings lassen sich bereits ab dem 18 . und 19. Jh. überdeutliche Hinweise darauf feststellen, dass die für heterodoxe Strömungen typischen Kennzeichen durch aus der Perspektive des normativen Islams unproblematische Elemente verdrängt werden: Es fällt auf, dass auf den zuletzt beigebrachten Beispielen auffällig häufig der Thronvers ( $\mathrm{Q}$ 2:255) kopiert wurde. Anstatt der unter dem Namen Šakl-i 'ayn-i 'ali bekannten Figur ${ }^{46}$ oder des Dū l-Faqār-Schwerts finden sich ab dem 18. und 19. Jh. auf den Rollen auch zunehmend Abbildungen von Blumen. Die soeben aufgezählten Elemente sind aus ideologischer Perspektive weniger verfänglich als dies auf die aus heterodox ausgerichteten Männerbünden bekannten Insignien zutrifft. Aus dieser Perspektive betrachtet kommt in den auf den Rollen über die Jahrhunderte feststellbaren Veränderungen einschlägiger Merkmale auch der Triumph des normativen Islams über die heterodoxen Strömungen zum Ausdruck. Dies ruft A. Knyshs Bemerkung in Erinnerung, dass sich der Niedergang der früher ausgesprochen reichen mystischen Tradition des Islams spätestens ab dem Anfang des 20. Jh. nicht mehr aufhalten liess und eigentlich schon deutlich früher eingesetzt hatte. ${ }^{47}$ Mit diesem Niedergang verliert die islamische Welt bedauerlicherweise auch eine Vielfalt und einen inneren Reichtum, die wesentlich zu ihrer Stärke beigetragen hatten.

\subsection{Exkurs: Dokument in Blockdruck-Technik: Khorasan, datiert 535/1140}

Diese Ausführungen machen im Sinn eines Exkurses auf ein im Jahr 535/1140 wohl in Khorasan im Blockdruck-Verfahren (Tarš-Technik) hergestelltes Exemplar aufmerksam. Es handelt sich um eine

46 Vgl. dazu Kapitel 6 (bei Anm. 123-124 und 139-184) und Nünlist, Rollen der Andacht aus dem Umfeld von Derwischorden 123-127.
Ergänzung zu Kapitel 7.2 (Anm. 2). Der Text wird aus der Beschreibung dieses Exemplars im Auktionskatalog von Christie's, London, übernommen. Dieser Katalog ist in öffentlichen Bibliotheken zumeist nicht verfügbar:48

„Christie's, London, King Street, Sale 7843, Art of the Islamic and Indian Worlds, 13 April 2010. A Seljuk talismanic scroll. Eastern Iran or Afghanistan, dated middle of Rabīi I AH 535/end of October 1140 AD.

Lot Description: [A Seljuk talismanic scroll] containing seven talismanic texts (hirz), Arabic manuscript on paper, comprising a long illuminated headpiece, the fatiha and seven further sections written in black cursory naskh, titles in kufic reserved on black cross-hatched ground within rectangular dotted frame, the headpiece starting from the shahada reserved on black ground and framed with the name Muhammad in geometric kufic, the central double-sided interleaved inscription with sura al-ikhlas and sura alkawthar in black and white foliated kufic on red cross-hatched ground issuing two compositions of three geometric stars within circular medallions, each medallion between fleshy palmettes, an almond shape medallion at top with floral arabesque issuing an intricate geometric design issuing the inscription nasr wa fath [ $\mathrm{min}$ Allah] in foliated kufic, undeciphered signature in the medallions, date at end possibly added, losses to top, waterstaining. $137 / 4 \times 41 / 8$ in. $(350 \times 10.5 \mathrm{~cm}$. $)$

Lot Notes: One inscribed medallion in the headpiece indicates that this scroll was written for a certain Alp Pahlavan.

This long scroll would have been carried tightly rolled and close to the body of its owner, probably in a small ornamented case. The presence of chosen Qur'anic suras and verses gives the talisman its magical power. Each chapter of this scroll groups under a specific title verses intending to protect

48 Diese Angaben aus http://www.christies.com/LotFinder/ lot_details.aspx?intObjectID $=5302917$ (Stand 20. Oktober 2017: ohne Abbildung; Stand 15. Oktober 2015: mit Abbildung); vgl. dazu Kapitel 7, Anm. 2. 
the owner against various misfortunes; it would have been used to avoid terror (faraq), famine (awja $),{ }^{49}$ deception (kid), the evil eye ('ayn), magic (sihr), and fear (khawf). The magical properties of these talismans were much diversified and their use was widely spread in the medieval Islamic world. As paper was relatively inexpensive, scrolls were amongst the most popular talismans.

The very long and intricate headpiece decorating this scroll, a third of the scroll's total length, is the most impressive feature of this manuscript. The inventiveness of the design is unique, playing with various geometric compositions based on the six-pointed star, the double-sided interleaved suras line in kufic script along the central cartouche, and the bold foliated kufic inscription at the extremity.

Beside this remarkable headpiece, the very early date of 1140 and the fact that the scroll has survived complete (excepted for its very top) confer on it an undeniable importance. Although nth century Fatimid stamped amulets have been found in Egypt, it has not been possible to compare this scroll with a similar contemporary or earlier example as there are probably a very small number of surviving examples (Trésors fatimides du Caire, exhibition catalogue, Paris, 1998, cat. 98-99). Later Mamluk Egypt however produced scrolls which follow the same organization of the headpiece: the oblong inscribed medallion is also bordered with medallions with six-pointed stars. Two richly illuminated scrolls, a complete Qur'an of the first half of the 14th century in the David Collection and a fragmentary talismanic scroll with Qur'anic verses dated circa 136o, show how much these scrolls were appreciated (Kjeld von Folsach, Art from the World of Islam in the David Collection, Copenhagen, 2001, cat. 9 and Islamic calligraphy, calligraphies islamiques, exhibition catalogue, Genève, 1988, cat. 22).

In her discussion of hexagons and hexagonal stars, Eva Baer indicates that this motif, sometimes

49 So in der Beschreibung in der in der vorangehenden Anmerkung angeführten Internetquelle. called Seal of Solomon, appears on 11th century perforated mosque lamps and becomes amongst the most popular design in metalwork from the early 12th century onward and is transferred from one material to another. The geometric medallions of a 12th to early 13th century penbox and a 12th century bucket in the LA Mayer Memorial Museum, the first attributed to Afghanistan and the second to Khorassan, closely relate to the strapwork visible on our scroll which support an Eastern Iran or Afghanistan attribution (Eva Baer, Metalwork in Medieval Islamic Art, New York, 1983, fig. 107-109, pp. 126-132).

An obvious comparable for the strapwork of the headpiece comes to mind in the slightly earlier slip-painted pottery vessels commonly attributed to Samanid Central Asia or Eastern Iran. The example offered in this sale (please see lot 10) gives a very close comparable to the decoration of our scroll. A bowl with abstract designs in the Kuwait National Museum displays similar palmettes to those found in the interstices between each of our medallions (Oliver Watson, Ceramics from Islamic Lands, London, 2004, p. 220). The fact that this group of ceramics is usually dated 1oth century is worthy of note. It is possible that our scroll is realized in a somewhat archaic style and therefore later than the period where the style flourished. However, the firm date of 1140 inscribed on this scroll forces us to reconsider the date of that particular group of so-called Samanid ware.

A rare Afghan hexagonal wooden table in the David Collection is painted and incised with similar palmettes and strapwork as that of our scroll (Kjeld von Folsach, op. cit., cat. 427). It is dated nth to 12 th century and supports the attribution of the scroll to the Eastern Iranian world. The inscriptions in kufic script found on the scroll can also be paralleled with examples on textile or manuscripts. The 11th to 12th century silk robe sold in these Rooms, 31 March 2009 (lot 94), has a calligraphic band with repeated interlocking letters between which grow sprays of fleshy tapering palmettes that closely relate to our example. A unusual Qur'an leaf in the Metropolitan Museum is inscri- 
bed with 6 lines of bold kufic with remarkably interlaced upstrokes, a number of them issuing palmettes with reserved designs similar to those of the scroll. Originating in Afghanistan, probably from Ghazna, this leaf was attributed to the middle of the 11th century (The Arts of Islam, Masterpieces from the Metropolitan Museum of Art, Berlin, 1981, cat. 16).

In many aspects, this scroll is an extraordinary example of mediaeval manuscript. Being a dated, heavily illuminated and comparable to numerous examples in other media, it is an essential piece to be included in the studies of 12th century works of art and manuscripts." 


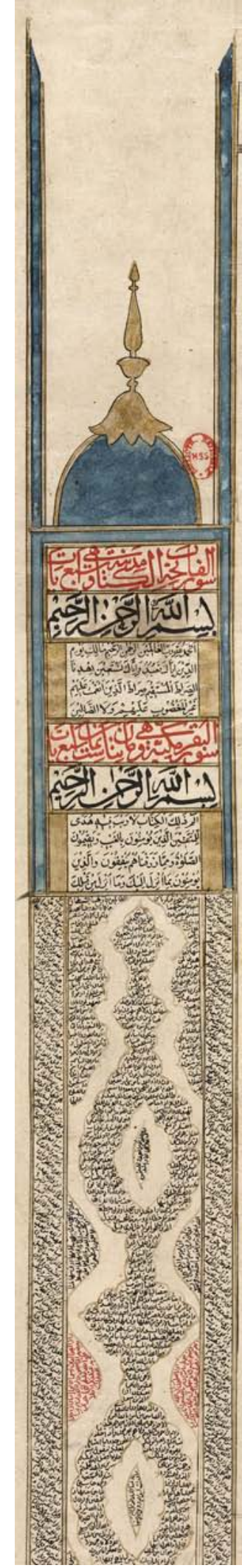

ABB. 161

Paris, Bibliothèque Nationale de France, Arabe 571, und Kopenhagen, Royal Library, Cod. Arab. Add. 49 (vgl. bei Anm. 10-15): Die Rolle Arabe 571 und ihr Parallelstück aus Kopenhagen (Cod. Arab. Add. 49) zählen zu den späten Dokumenten (wohl 18. Jh.). Arabe 571 ist am Anfang vollständig erhalten. Das Anfangszierfeld zeigt eine blaue Moscheekuppel mit goldenem Aufsatz. Unmittelbar darunter die hervorgehobenen Abschriften von Suren 1 und 2.

ARABE 571 (BIBLIOTHÈQUE NATIONALE DE FRANCE, PARIS) 


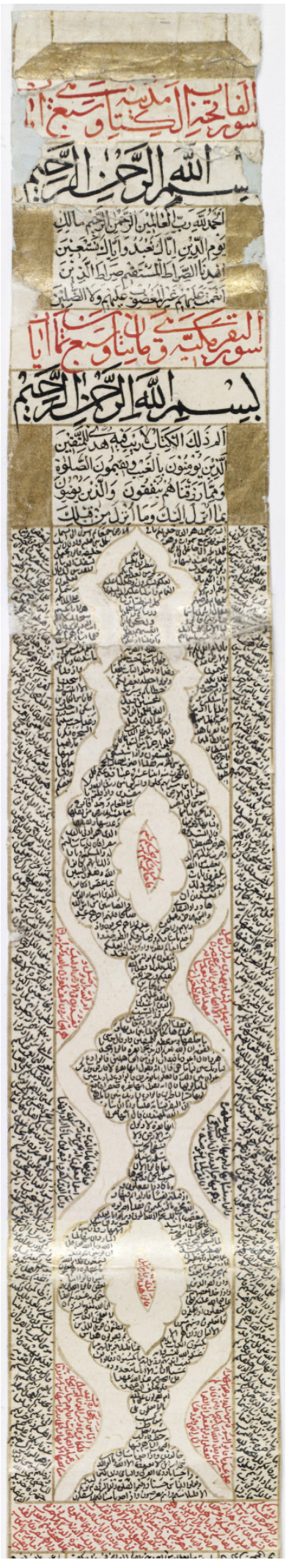

ABB. 162

Paris, Bibliothèque Nationale de France, Arabe 571, und Kopenhagen, Royal Library, Cod. Arab. Add. 49 (vgl. Abschnitte zwischen Anm. 15 und 17): Anfang des Hauptteils der Rolle, auf dem eine säulenförmige Verzierung auffällt, die an die Aufsätze auf hinduistischen oder buddhistischen Sakralbauten in Indien erinnert (hier der Anfang der Rolle aus Kopenhagen). 


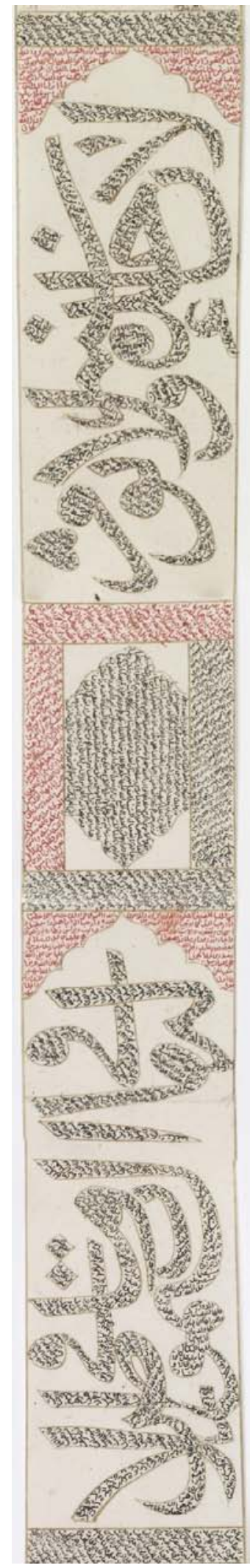

ABB. 163

Paris, Bibliothèque Nationale de France, Arabe 571, und Kopenhagen, Royal Library, Cod. Arab. Add. 49 (vgl. nach Anm. 17): Auf Cod. Arab. Add. 49 und Arabe 571 ist auf zwölf Textfelder verteilt der Thronvers (Q 2:255) kopiert worden. Dieser grosse Text wird durch kleinen Text in $\dot{G} u b \bar{r} r$ gebildet. Die Texteinteilung stimmt auf beiden Rollen überein. Hier gezeigt die Abschnitte mit der Aussage Lā ta'hudu-hū sinatun wa-lā nawmun (Textfeld IV) und la-hū mā fi s-samawāt wa-mā fi l-ard (Textfeld v; Rolle aus Kopenhagen).

COD. ARAB. ADD. 49 (ROYAL LIBRARY, KOPENHAGEN) 


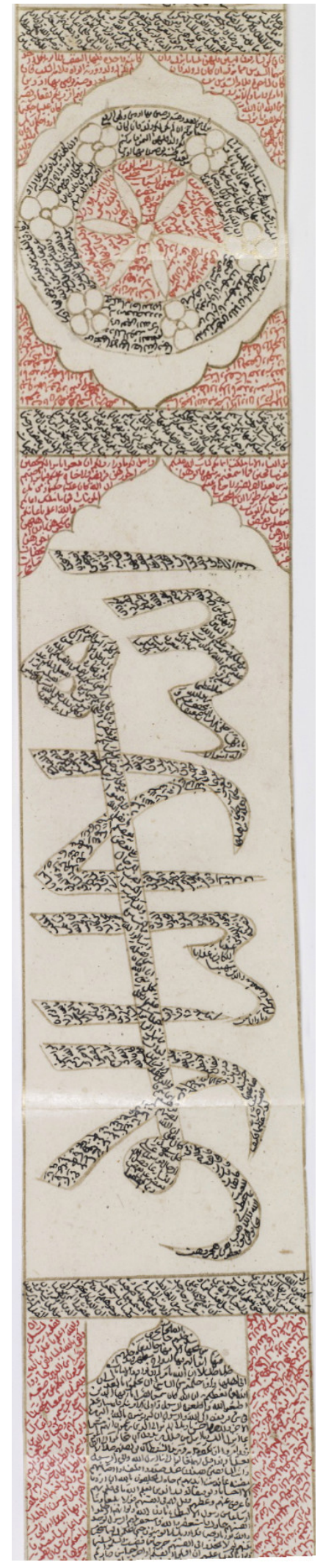

ABB. 164

Paris, Bibliothèque Nationale de France, Arabe 571, und Kopenhagen, Royal Library, Cod. Arab. Add. 49 (vgl. Abschnitt mit Anm. 20): Abbildung eines kreisförmigen Zierelements, das auf Cod. Arab. Add. 49 zwischen Textfeld I und II (Allāhu lā ilāha illā huwa) angebracht worden ist. Auf dem Pariser Dokument fehlen vergleichbare Verzierungen.

COD. ARAB. ADD. 49 (ROYAL LIBRARY, KOPENHAGEN) 

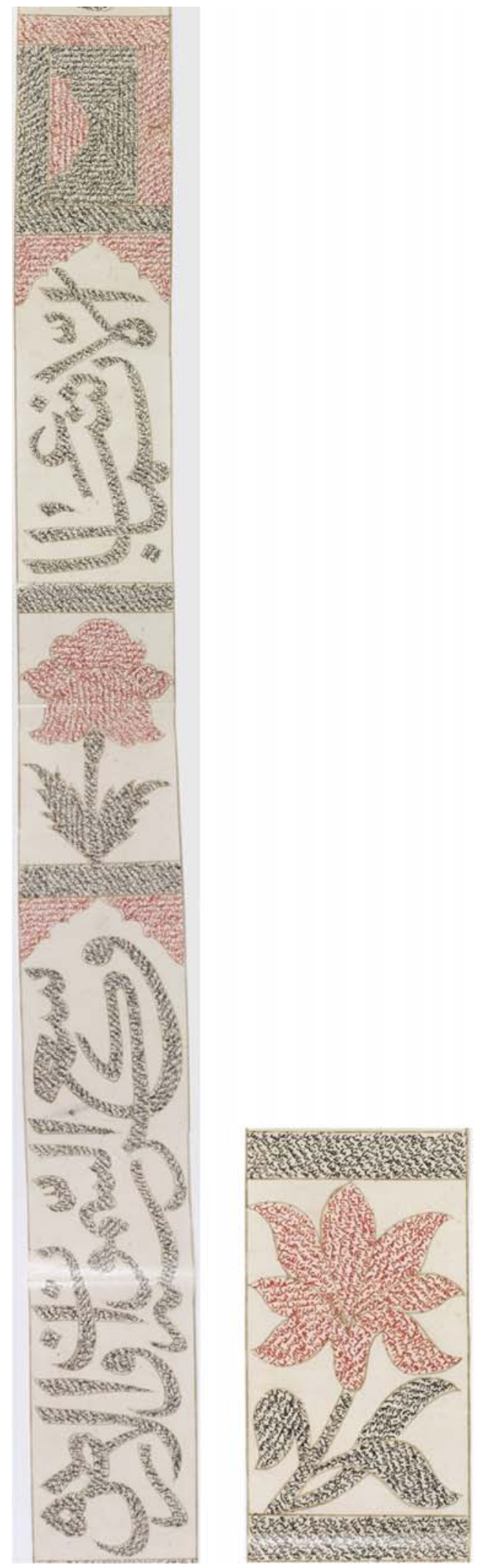

ABB. 165

Paris, Bibliothèque Nationale de France, Arabe 571, und Kopenhagen, Royal Library, Cod. Arab. Add. 49 (vgl. Abschnitt mit Anm. 21): Auf späten Rollen wurden oft Verzierungen in Form von Blumen angebracht. Hier gezeigt eine Blume zwischen Textfeld x (illā bi$\left.m \bar{a} s^{s} \bar{a} a\right)$ und XI (wasi'a kursiyyu-hū as-samawāt wa-l-ard) auf der Rolle aus Kopenhagen. Diese Blumen werden durch Textelemente in $\dot{G} u b \bar{a} r$-Schrift gebildet (Stellen aus dem Koran). Die Blüte selbst ist rot, ihr Stiel schwarz. Auf der Rolle aus Paris fehlen derartige Verzierungen.

COD. ARAB. ADD. 49 (ROYAL LIBRARY, KOPENHAGEN) 